with Parkinson's disease psychosis (PDP). Depression affects up to $60 \%$ ofPD patients and is frequently treated with SSRIs/SNRIs. Data suggest the potential for a synergistic effect between 5 HT2A receptor inverse agonist/antagonists and SSRIs insubjects with neuropsychiatric disease. This post-hoc analysis evaluated a subgroup of subjects from the pimavanserin clinical program to determine if there was any difference in antipsychotic response between the subjects receiving pimavanserin in combination with an SSRI versus those without.

METHOD: A pooled analysis of two 6-week randomized, double-blind, placebo-controlled Phase 3 studies was conducted to assess the overall treatment effect of pimavanserin34 mg. The outside-North America region in Study 012 was not included due to a difference in methodology in the assessment of the primary endpoint. Subjects in both the 020 and 012 studies received 42 days of treatment. The mITT population included 268 subjects; with 135 in the pimavanserin group. The full safety dataset included 433 subjects; with 202 in the pimavanserin group. Of the 268 subjects in the mITT population, a total of 77 received concomitant therapy with SSRIs. A subgroup analysis was conducted to determine if there was any difference in response among the subjects receiving concomitant SSRIs.

RESULTS: Overall, pimavanserin demonstrated a -6.21point improvement in psychosis at Week 6 as measured by the PD-adapted Scale for Assessment of Positive Symptoms (primary change from baseline analysis [MMRM]). The treatment difference was 2.87 points over placebo $(\mathrm{p}<0.001)$ and was clinically meaningful. Both subgroups (pimavanserin +/- SSRI) demonstrated a statistically significant improvement over placebo. Among subjects taking concomitant SSRIs, the decrease in psychosis symptoms was more prominent for both pimavanserin and placebo-treated subjects (-8.33 points and -4.01 points, respectively) compared to the 189 subjects not taking SSRIs (-5.36 points and -3.01 points, respectively); the treatment difference was of greater magnitude in the concomitant SSRI treated group (-4.32 vs. -2.34$)$. A total of $10 \%(4 / 40)$ and $7.4 \%$ $(12 / 162)$ of pimavanserin treated subjects, with and without SSRIs, respectively, discontinued because of adverse reactions.

CONCLUSIONS: The results of this analysis further support findings that the combination of selective 5-HT2A agonist/ antagonists and SSRIs may have additive beneficial effects, suggesting a possible enhancement of antipsychotic effect in subjects treated with concomitant SSRIs.

FUNDING ACKNOWLEDGEMENTS: Clinical study was funded by ACADIA Pharmaceuticals Inc.
136

\section{CerefolinNAC Therapy-Induced Dizziness}

Jasir T. Nayati, CNMT'; and Alan R. Hirsch, $M D^{2}$

${ }^{1}$ St. James School Of Medicine, Park Ridge, IL /

Anguilla Campus

${ }^{2}$ Smell and Taste Treatment and Research Foundation, Chicago, IL

ABSTRACT: Study Objective: CerefolinNAC (CFLN-NAC) contains L-methylfolate (6 mg), methylcobalamin (2 mg), and N-acetylcysteine [NAC] $(600 \mathrm{mg})$ [Pamlab 2017]. Dizziness and lightheadedness have not heretofore been described with use of CFLN-NAC.

METHODS: Case Study: A 64 year old right-handed female was started on CFLN-NAC for smell and taste issues. Over a three day period, she experienced a gradual increase in dizziness. This was a non-vertiginous lightheadedness, so severe that she was unable to walk, and would lie down the entire day to alleviate the dizziness. It was associated with nausea, but without any vomiting or falls. The dizziness would come and go, last for several hours, and was $9 / 10$ in severity.

She admits to a past history of epochs of vertigo. The vertigo occurred three times with nausea and vomiting 13 years, 11 years, and 4 years prior to presentation. She also developed a constant, bilateral, high-pitched tinnitus 14 years prior to presentation, which obstructs her hearing. It is level $3 / 10$ in intensity during the night and in the quiet. There were no alleviating or aggravating factors. Acupuncture was without effect, and she denies any ear pain. After ceasing CFLN-NAC for three days, a gradual reduction of dizziness to baseline ensued.

RESULTS: Abnormalities in Physical Examination: General: Decreased blink frequency and hypokinesia. Cranial Nerve III, IV, VI: Saccadization of horizontal eye movements. Motor Examination: Tone: 1+ cogwheel rigidity in both upper extremities, left more than right. Drift Test: Bilateral Abductor Digiti Minimi sign with cerebellar spooning. Reflexes: Absent quadriceps femoris and Achilles bilaterally. Positive Hoffman reflex bilaterally. Neuropsychiatric Testing: Go-No-Go Test: 6/6 (normal). Animal Fluency Test: 19 (normal). Reliable Digit Span: 10 (normal). Clock Drawing Test: 4 (normal). Center for Neurologic Study Lability Scale: 10 (normal). Other: Audiometry and Fiberoptic Endoscopy: normal MRI of the brain with and without contrast was normal.

CONCLUSION: None of the individual components in CFLN-NAC have been reported to precipitate dizziness [Pamlab 2017]. The non-vertiginous nature of the dizziness makes it unlikely to be due to vestibular involvement, raising the spectre of this drug having an impact on the autonomic nervous system. While a 
nocebo effect could be in action, this is unlikely since dizziness was not presented as a potential side effect on initiation of the medication. In addition, methylcobalamin can cause hyperviscosity syndrome, but due to an absence of visual disturbances and altered mental status, it is also improbable. The relatively rapid onset with initiation and resolution upon discontinuation of this medication strongly suggests that it is not a coincidence, rather an origin for the dizziness. Those who are treated with CFLN-NAC should be queried as to new onset dizziness. For those already dizzy, one should consider other treatment options.

Funding ACKNOWLEDgemEnTS: Smell and Taste Treatment and Research Foundation

\section{7}

\section{Menstrual Synchrony of Burning Mouth Syndrome}

Jasir T. Nayati, CNMT' ; and Alan R. Hirsch, $M D^{2}$

${ }^{1}$ St. James School Of Medicine, Park Ridge, IL /

Anguilla Campus

${ }^{2}$ Smell and Taste Treatment and Research Foundation, Chicago, IL

ABSTRACT: Study Objective: Burning mouth syndrome (BMS) is characterized by oral mucosal burning sensations, with normal clinical and laboratory results. Menstrual synchrony of migraines and epilepsy have been discussed; however, menstrual synchrony of BMS has not heretofore been described.

METHODS: Case Study: A 29 year old right-handed female exhibited intermittent BMS symptoms, one month after suffering a left parietal infarction. She describes the pain as a burningsensation, localized to the bilateral and anterior aspects of her tongue. It lasts for four days, starts three days prior to her menses, and occurs twice a month. She is unable to correlate any patterns or triggers that may cause to exacerbate her BMS. She denies any taste disturbances, hot-flashes, night sweats, and perspiration.

RESULTS: Abnormalities during neurological examination were noted. Cranial nerves (CN) III, IV, and VI showed bilateral lateral first degree end-gaze unsustained nystagmus. CN IX and X showed decreased bilateral gag reflex. A right pronator drift with a right abductor digiti minimi sign was seen in the motor examination. The cerebellar examination was positive for bilateral dysmetria during the Finger-To-Nose examination, and exhibited Holmes rebound phenomena, right more than left. Sensory examination showed decreased light touch in the lower extremities, right more than left. Hoffman reflex was bilaterally positive. Mental status examinations demonstrated poor similarity interpretation and calculation ability. Her neuropsychiatric testing was normal, and included the Go-No-Go and Animal Fluency Testing. MRI of the brain exhibited gliosis/laminar necrosis in the left inferior parietal lobe, and an $8 \mathrm{~mm}$ descent of cerebellar tonsils below the foramen magnum.

CONCLUSION: The potential mechanism for catamenial BMS is manyfold. Estrogen and progesterone both have nociceptive properties. Premenstrual drop or reduction of estrogen and progesterone may act to disinhibit pain [Vincent 2008], with pain modulation being more effective during the ovulatory phase (high estrogen and low progesterone) [Rezaii 2012]. Depression in the presence of Late Luteal Phase Dysphoric Disorder may function to exacerbate the perception of underlying pain throughout the body, including the mouth and tongue. Decrease in estrogen and progesterone levels may also alter salivary output and composition. This may allow baseline reduction of proprioceptive input on the tongue, thus acting through Melzack and Wall's Gate Control Theory of Pain to disinhibit small C fibers, which is perceived as burning pain [Melzack 1978]. Along with menses, olfactory ability drops, and food preferences are often reported to change [Keller 2013]. A decrease in estrogen and progesterone can also enhance trigeminal nerve sensitivity [Martin 2007], which exacerbates pain. This may indirectly influence or be associated with her BMS. Such observations justifies a trial of hormonal agents for therapy of BMS.

FUNDING ACKNOWLEDGEMENTS: Smell and Taste Treatment and Research Foundation

\section{8}

\section{Gait Ignition Failure Syndrome Secondary to Spinal Stenosis}

Jasir T. Nayati, CNMT'; Angela Rekhi ${ }^{2}$; and Alan R. Hirsch, $M D^{3}$

${ }^{1}$ St. James School Of Medicine, Park Ridge, IL/

Anguilla Campus

${ }^{2}$ Aureus University School of Medicine, Fairlawn, NJ/ Aruba Campus

${ }^{3}$ Smell and Taste Treatment and Research Foundation, Chicago, IL

ABSTRACT: Case Objective: Gait ignition failure syndrome, where immobility occurs only upon initiation of ambulation and normal gait ensues once entrained, has been reported with frontal lobe and midbrain locomotor region pathology. However, gait ignition failure 